\title{
Refining Genetic Algorithm Twin Removal for High-Resolution Protein Structure Prediction
}

\author{
Trent Higgs*, Bela Stantic*, Md Tamjidul Hoque ${ }^{\dagger}$, Abdul Sattar*‡ \\ ${ }^{*}$ Institute for Integrated and Intelligent Systems (IIIS), \\ Griffith University, Queensland, Australia \\ Email: \{T.Higgs, B.Stantic, A.Sattar\}@griffith.edu.au \\ ${ }^{\dagger}$ School of Informatics, Indiana Center for Computational Biology and Bioinformatics, \\ Indiana University Purdue University Indianapolis (IUPUI), USA \\ Email: Tamjidul.Hoque@gmail.com \\ ${ }_{\ddagger}^{\ddagger}$ NICTA Queensland Research Lab
}

\begin{abstract}
To gain a better understanding of how proteins function a process known as protein structure prediction (PSP) is carried out. However, experimental PSP methods, such as $X$-ray crystallography and Nuclear Magnetic Resonance (NMR), can be time-consuming and inaccurate. This has given rise to numerous computational PSP approaches to try and elicit a protein's three-dimensional conformation. A popular PSP search strategy is Genetic Algorithms (GA). GAs allow for a generic search approach, which can provide a generic improvement to alleviate the need to redefine the search strategies for separate sequences. Though GA's working principles are remarkable, a serious problem that is inherent in the GA search process is the growth of twins or identical chromosomes. Therefore, enhanced twin removal strategies are crucial for any GA search solving hard-optimisation problems like PSP. In this paper we explain our high-resolution GA feature-based resampling PSP approach and propose a twin removal strategy to further enhance its prediction accuracy. This includes investigating the optimal chromosome correlation factor (CCF) for our approach and defining a pre-built structure library for twin removal. We have also compared our GA approach with the popular Monte Carlo (MC) method for PSP. Our results indicate that out of all the CCF values we tested a CCF value of 0.8 provided the best level of diversity within our GA population. It also generated, on average, more native-like structures than any of the other CCF values, and clearly demonstrated that twin removal is needed in PSP when using GAs to obtain more accurate results.
\end{abstract}

\section{INTRODUCTION}

Proteins are important macromolecules that perform biological tasks on a cellular level. A protein is formed by a string of amino acids folding into a specific three-dimensional shape, which determines the biological task it will perform. These biological tasks are quite significant in the biology domain, and by determining their final three-dimensional structure more insight into their function can be elicited. To do this a process known as protein structure prediction (PSP) is carried out.

PSP can be a very time consuming task. Therefore, computational PSP methods have been proposed. These methods can be grouped into three main categories: comparative modelling, threading, and ab initio. Ab initio, the most difficult out of the three, is based on Afinsen's 'Thermodynamic Hypothesis' [1]. This states that a protein's native structure is at its lowest free energy minimum.

The $a b$ initio method in PSP has been looked at from many different perspectives, and therefore has a large amount of work utilising different search approaches. These include numerous versions of Monte Carlo (MC), [2], Ant Colony Optimisation [3], and Simulated Annealing (SA) [4]. Statistical approaches such as Chain Growth (CG) [5], and Contact Interaction (CI) [6] have also been developed. However, most of these search approaches have the problem that as the sequence length increases the accuracy of the predicted structure decreases. Despite this, further research into bioinspired algorithms [7], [8], such as Genetic Algorithms, have proven to be quite promising in solving the PSP problem [9], [10].

GAs are particularly useful as they can be applied to a wider range of problems that are in their generic forms, which often alleviates the need to redefine the search strategies for each separate sequence. A technique that can be applied to GAs is feature-based resampling, which is a way of refining local minima from a previous search space [11]. It works by introducing the concept that even if most of the structures generated for a target protein (i.e. decoys) from the previous search are not close to the native conformation, they still may contain native-like features that when combined together will create protein structures that are closer to the native conformation.

Structural search algorithms, like GAs, are quite popular because of their crossover and mutation operators. The crossover operator is the heart of the GA search, and has been adapted in numerous other non-deterministic search approaches [12]. Despite being effective, GAs like many other search techniques can stall. The main factor that causes stalling in GAs is the faster growth of identical or highly-correlated chromosomes: as the GA search proceeds the similarity within the population grows, which can cause twins or identical chromosomes to appear.

In this paper we present a method of refining twin removal for high-resolution PSP using our GA feature-based resampling approach. Removing twins was carried out by performing experiments to elicit the optimal chromosome correlation 
factor (CCF) [13], defining a upper and lower bound for the root mean square deviation (RMSD) structural measure, and using a structure library to fill the gaps of removed twins within the population. To further highlight the successfulness of our GA method we have also implemented a Monte Carlo PSP approach to compare our results against.

The remainder of this paper is organised as follows. In Section 2 the background is discussed, Section 3 outlines the methodology we have applied, Section 4 explains the experimental setup, Section 5 presents and discusses the results we gained from our experimentation, and in Section 6 we draw our conclusions.

\section{BACKGROUND}

Computational protein structure prediction (PSP) approaches mainly use search techniques to determine the global minimum of an energy function, except for comparative modelling (which works by finding homologous conformations within protein structure databases). All other methods (e.g. threading and $a b$ initio) search the energy landscape of a protein's conformational space to find a global minimum of the energy function. Two popular methods in the literature that accomplish this are Monte Carlo (MC) based algorithms [14] and Genetic Algorithms (GA) [9].

MC algorithms for PSP problems work by conducting a random walk of the energy landscape. It only accepts a new state if the energy is lower than the current state (i.e. gradient descent). The problem that MC methods have, like numerous other gradient descent algorithms, is that it can easily get trapped in local minima. The technique that is applied to alleviate this problem is Metropolis criterion [15]. Metropolis MC algorithms use a Boltzmann equation. This equation contains a temperature variable, which when increased makes the probability of accepting neighbouring higher energy states more likely.

The GA search approach, in comparison, is evolutionary in nature. It starts off with a large pool of genetic traits, which by use of genetic operators are reproduced, sometimes with random mutations to escape local minima, and are subjected to natural selection (i.e. the fittest survives). By doing this, there is no guarantee that the absolute best solution will be found, however it is apparent that over time it creates solutions that contain a combination of genetic traits, which function better in its defined environment guided by the fitness function $f$. In PSP there has been a large amount of work in GAs using low-resolution models [9], [10], [16], and very limited work using high-resolution models [17], [18].

Feature-based resampling techniques can easily be applied to GAs that have been developed for PSP applications. It revolves around using native-like features from the previous sampling round. If no models from the previous round of sampling produces a structure close enough with the native structure, they still may contain various native-like features, which can be recombined to create new structures that are closer to the native conformation. This is analogous with the crossover operator within GAs. For example, protein features from a previous generation are combined together in the crossover operator to create more native-like structures to be carried over into the next generation.

A severe condition that GAs suffer from is that they can stall due to the population growing in similarity [19], [13]. This is caused by twins or identical chromosomes entering the population, and cutting down the effectiveness of the crossover operator. For example, using a simplistic case, twins that are selected for breeding will produce offsprings that are identical to the parents, and therefore add more of the same genetic material into the population at the evaluation stage. In regards to PSP, this is quite a significant problem as it can take a large number of iterations to converge compared to less complex problems. For this reason it is important to gain an understanding of this condition in the GA search so that more efficient techniques can be developed to solve the PSP problem.

Twins and methods to remove them are not new concepts as far as GAs are concerned. Haupt proposed initialising each chromosome in the initial population with a different pattern to minimise twin growth [20]. However, twins occur due to the GA search process, therefore any individuality gained by this process would steadily decline after each generation. A quite popular approach is to not allow twins to breed with each other [21]. The main problem with this approach is as the GA search proceeds, and twins continue to grow, it becomes very costly to find dissimilar chromosomes to mate with in the population. Another method used to enforce diversity within a GAs population is the niched-penalty [22]. This works by imposing a penalty on any group of chromosomes that have a specific similarity with each other to discourage them participating in the next generation.

From the related work above [20], [22], [13] we have shown that twins are a inherent problem in the GA search approach. Therefore, we will apply techniques that have been highlighted in [19] and [13] to determine the chromosome correlation factor (CCF) value that provides an optimal level of diversity in the high-resolution PSP problem, which should guide our GA to more accurate predictions. This will also be accompanied by a comparison against a Monte Carlo (MC) based approach to show the successfulness of our method.

\section{Methodology}

Genetic Algorithms (GA) are very susceptible to the appearance of twins within their population, which can cause the search to stall or halt, and produce sub-optimal solutions. In the next three sections we will explain our GA feature based resampling algorithm, outline the MC approach we have developed for comparison, and redefine the twin removal process so it can be applied to our high-resolution GA approach.

\section{A. GA Feature-Based Resampling Approach}

Our feature-based resampling GA [23] works by taking the initial predicted structures from a complete run of a protein structure prediction (PSP) software using an arbitrary target protein. These initial structures are then used as input into our 


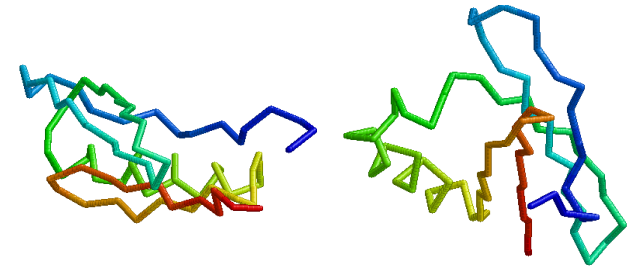

(a) Parent 1

(b) Parent 2

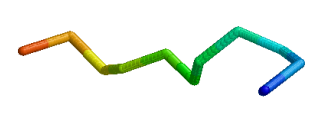

(c) Feature 1

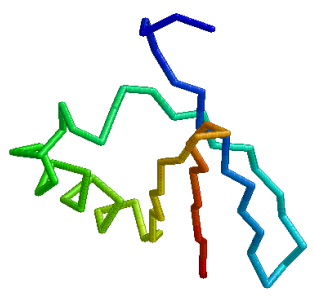

(d) Feature 2

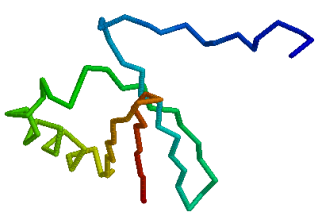

(e) Offspring

Fig. 1: An example of the crossover operator using the protein $2 \mathrm{ptl}$ where $n=13$. The first 12 residues of parent 1 (a), are joined with the remaining 65 residues in parent 2 (b). From this you can see that the first feature is made up of residues 1-12 in parent 1 (c), and the second feature is made up of residues 13-78 (d), which when joined together form the offspring depicted in (e).

GA for refinement. The following four sections will outline our GAs main search operators.

1) Selection: Selection in the GA search is very important. It dictates which chromosomes are selected for breeding in the crossover operator. For our GA we have chosen one of the most common approaches: the roulette wheel, which is based on proportionate selection (see Equation 2).

In our algorithm we have allowed a probability of $80 \%$ to select chromosomes with medium to high $f$, and a $20 \%$ probability to select chromosomes with medium to low $f$. These values were chosen to allow fitter chromosomes to be selected most of the time, but also to give a small chance to chromosomes with smaller fitness values to be selected as they may contain features that could improve the search.

2) Crossover: The crossover operator is the heart of the Genetic Algorithm (GA). In our high-resolution approach we have modelled the crossover operator off of Unger's low-resolution PSP method [9]. We store our structures $S$ of length $m$ as a set of Euclidean points $S\left(a_{1(x, y, z)}, a_{2(x, y, z)}, \ldots, a_{m-1(x, y, z)}, a_{m(x, y, z)}\right)$, and crossover is carried out by splicing two protein structures together using a randomly selected position in the chain and then exchanging the sliced parts. As we are dealing with a more detailed model than Unger we had to decide which position/s created structures with the least amount of clashes when crossover was taking place. We found that the $C_{\alpha}$ atom provided us with the best results in this regard (i.e. less collisions and infeasible structures), therefore we have limited our crossover points to be $C_{\alpha}$ atoms only.

3) Mutation: Mutation is used to stop the GA from getting trapped in local minima and stalling. There are numerous ways of doing this, but one of the main techniques is to use rotational moves to mutate a protein's conformation [9] [10]. In our approach we have used pivot rotation moves on the $x, y$, and $z$ axis to mutate protein structures. As with the crossover operator, and for the same reason, we have limited mutation points to $C_{\alpha}$ atoms only. Pivot rotations work by translating all points to a chosen pivot $(n)$ and rotating the sub-structure around that pivot point $(n+1$ to $m)$. The sub-structure, in this case, refers to all the points in a protein structure from $n+1$ to the end of the structure $(m)$. An example of a pivot rotation, using a backbone representation of the protein chain can be found in Figure 2.

4) Scoring Methods: To score the final output of our GA, and compare it against the original decoys, two structural similarity measures are used. These are root mean square deviation (RMSD) [24], and template modelling score (TM-Score) [25]. RMSD works by summing the Euclidean distances (e.g. $R M S D(v, w)=\sqrt{\frac{1}{n} \sum_{i=1}\left\|v_{i}-w_{i}\right\|^{2}}$ ) between every residue from the two structures it is trying to compare. TMScore, in comparison, is more sensitive to close matches than distant matches (unlike RMSD). This means if one part of the protein is completely wrong, but the other topology is quite similar the TM-Score will not be as drastically effected.

\section{B. Application of MC for PSP problem}

Most Monte Carlo (MC) approaches [15] apply random variance to improve a solution. It starts with a random conformation $S_{1}$ with energy $E_{1}$. It then applies some random change to the conformation to make a new solution $S_{2}$, which has energy $E_{2}$. If the energy improves $\left(E_{2}<E_{1}\right)$ then accept the change, otherwise the metropolis criterion (see Equation 1) is applied to decide whether or not to accept the change, which came about from the simulated annealing search technique.

$$
r<\exp \left[\frac{E_{1}-E_{2}}{T}\right]
$$

In Equation $1 r$ refers to a random number between 0 and 1 , and $T$ refers to the temperature of the system, which is cooled throughout the search to obtain convergence. In our approach we have used similar techniques that were applied in our GAs mutation operator to allow for a fair comparison of the two algorithms. Therefore, the random change in the MC algorithm will be conducted by using rotational move sets. Our temperature will be 2 , and cooled at 0.00000495 per MC step [9].

\section{Redefinition of Twin Removal}

Twins are a common occurrence in the GA search process. This is due to the selection procedure, favouring the fitter chromosomes to breed more frequently and hence resulting 


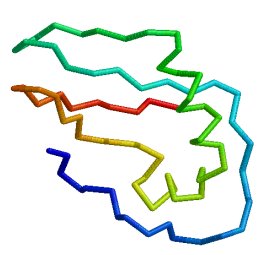

(a) Original

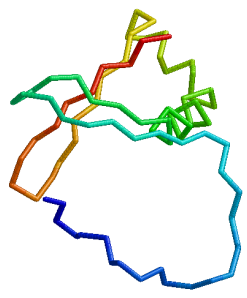

(b) Mutated
Fig. 2: An example of pivot rotation using the protein 2ptl, where $n=31$. Residue 31 is randomly selected to be the pivot $(n)$. The substructure (from residue $\#(n+1)$ to $\# \mathrm{~m}$ ) is rotated $90^{\circ}$ on the $x$ axis, which gives us the structure in (b).

in increasing similarities among chromosomes in newer generations. For example, given Equation 2 and a population of 8 chromosomes with $f: 8,6,7,9,1,4,11$, and 18 respectively. If the selection probability of a Chromosome $\mathrm{C}_{i}$ is $p_{i}$, then $p_{1}$ is $8 / 64, p_{2}$ is $6 / 64, p_{3}$ is $7 / 64$ and so forth. Now consider a population containing 8 chromosomes with $f: 8,4,4,4,4,4,3$, and 2 respectively, and all chromosomes with the same fitness are the same conformation (i.e. $C_{2}=C_{3}=C_{4}=C_{5}=C_{6}$ ). Therefore, the same result will be achieved if any of these chromosomes are selected for crossover or mutation operations.

$$
p_{i}=\frac{f_{i}}{\sum_{j=1}^{N} f_{j}}
$$

The problem, as highlighted in the above example, is that the probability of selecting a chromosome where $f=4$ is $60.6 \%$. So it can be seen that the effective selection probabilities for $C_{1},\left(C_{2}, C_{3}, C_{4}, C_{5}, C_{6}\right), C_{7}$, and $C_{8}$ would be $8 / 33,20 / 33$, $3 / 33$, and $2 / 33$ respectively. This means that the probability of selecting a chromosome where $f=4$ is much higher than a chromosome where $f=8$, which is not reflected in Equation 2 , and facilitates the propagation of twins in the GA search process.

To remedy the occurrence of twins the approach in [19] proposed the idea of removing only identical chromosomes from the population. However, it was shown in [13] that not only identical chromosomes were a problem, but also highlycorrelated chromosomes (i.e. having a similarity of $80 \%$ or more). This was only tested on the low-resolution model and no experimentation on the high-resolution model was provided. Therefore, we will extend these ideas into our approach for the high-resolution PSP problem, and carry out an experiment which identifies the percentage of twins removed using different chromosome correlation factors (CCF) to identify the optimal settings for our high-resolution approach. CCF is a measure of similarity between two chromosomes from 0 to 1. If a chromosome has an $>=80 \%$ similarity with another chromosome, their CCF factor would be 0.8 .

The other extension we have included is a structure library for twin replacement. When twin-chromosomes are removed from the population gaps appear, therefore an approach to replace these gaps is required. As we are using a featurebased resampling approach we have developed a structure library of local minima from the PSP software that was used to create the initial population to replace twins with. Meaning the structure library we are using is a collection of pre-built folding instances from the protein sequence that is being predicted. Our reasons for doing this is that the use of structure libraries in PSP have produced promising results in creating accurate predictions [14], [26], the PSP process is not slowed down, and time can also be saved by doing the prediction and structure library in parallel (note that this has not been implemented for this paper).

Replacements for twins are pulled out of the structure library at random. If the structure library for any reason runs out of protein conformations we create a new structure library based on the previous one. The reason for this is that regardless of how many protein conformations are kept in the structure library, eventually they may run out depending on how many twins are being replaced each generation, unless the structure library is created in parallel with the PSP process. Our solution for this is once the structure library is empty we take the original structure library and apply random mutations to the protein conformations contained within it, and add them back to the structure library. By doing this, we are still using structures that have some relation to the target protein and contain new genetic material for the GA search.

To carry out twin removal a way of defining the similarity between two structures is required. For simplicity sake we have chosen to use the root mean square deviation (RMSD) measure, which is discussed in section III-A. To define a CCF for this measure we have chosen to make the range 0-100, meaning 0 is identical, and 100 is completely different. As there is no definition for $100 \%$ dissimilarity with RMSD we have chosen a value that represents a large difference between two protein structures, which is 100 . Therefore, if a CCF of 0.8 is defined (i.e. $>=80 \%$ similarity), this corresponds to $<=20$ RMSD cut off value between two chromosomes in the population $(100-(0.8 * 100)=20 \%$ RMSD similarity $)$. When a replacement is made the chromosome with the better fitness is kept.

\section{EXPERIMENTAL SETUP}

All experiments, for our feature-based resampling GA, were conducted using the same set up as defined in [23]. In regards to our MC approach, we tried to keep the same amount of operations between the two algorithms to make it a fair comparison. To do this we calculated the amount of operations caused by the crossover operator $(100 * 200 * 0.7)$ and the amount of operations performed by our mutation operator $(100 * 200 * 0.1)$, and added them together together to gain the number of steps used for each MC simulation (i.e. 16000 steps). All the proteins we used for both our GA and MC experiments consisted of only one chain.

Finally CCF values will start at 0.4. The reason for this is that results gained from 0.4 were poor, therefore going any 
TABLE I: Results obtained from the initial Rosetta run, before resampling is applied.

\begin{tabular}{lrrrr}
\hline Protein & Length & $f$ & $R M S D$ & TM-Score \\
\hline 2ptl & 78 & -124.10 & $8.764 \AA$ & 0.4084 \\
1pgx & 83 & -120.12 & $4.385 \AA$ & 0.6570 \\
1bds & 43 & -21.97 & $6.151 \AA$ & 0.2138 \\
$1 \mathrm{bm} 8$ & 99 & -82.09 & $7.814 \AA$ & 0.2751 \\
$1 \mathrm{emw}$ & 88 & -48.36 & $8.415 \AA$ & 0.2856 \\
1 aoy & 78 & -57.80 & $6.028 \AA$ & 0.3854 \\
$1 \mathrm{csp}$ & 67 & -84.95 & $2.576 \AA$ & 0.7156 \\
$2 \mathrm{ppp}$ & 107 & -27.43 & $9.632 \AA$ & 0.2780 \\
$1 \mathrm{kjs}$ & 74 & -42.35 & $4.541 \AA$ & 0.4706 \\
1 vcc & 77 & -70.08 & $2.950 \AA$ & 0.6800 \\
\hline
\end{tabular}

lower was not required. The interval between CCF values we have chosen is 0.2 (e.g. 0.4, 0.6, 0.8, 1, and WT (without twin removal)). This interval was chosen for two reasons: (1) time constraints, each test takes an ample amount of time to complete, and (2) a data spread of 0.2 should be able to indicate which value/s provide better results or solutions.

\section{EMPIRICAL RESULTS}

Table I contains the best predicted structures for a specific target protein produced by Rosetta using ab initio protocols. These results are the benchmarks for our improvement values, as each structure belongs to a set of local minima from the same target protein that was used as the initial population to our GA search. This table highlights the protein's fitness score $(f)$, RMSD value, and TM-Score value.

Table II contains the results from our GA approach using different CCF values. All improvement values (columns 6 and 7) are based on the benchmarks presented in Table I. Figure 3 a depicts the average twin removal percentage for a particular chromosome correlation factor (CCF). The $x$ axis is the CCF value, and the $y$ axis is the removal percentage. Figure $3 \mathrm{~b}$ shows the average improvement for both RMSD and TMScore for a particular CCF value using the improvement values presented in Table II. Finally Figure $4 \mathrm{a}$ and Figure $4 \mathrm{~b}$ provide a comparison between our GA and MC methods.

\section{A. Analysis and Discussion}

To gain an understanding of what twin removal threshold (i.e. CCF) would perform the best for our feature-based GA approach we evaluated how many twins on average were removed for a given chromosome correlation factor (CCF). Note that all CCF values refer to $>=C C F$ value for twin removal purposes. For example if twin removal is based on $80 \%$ similarity, which gives us a CCF of 0.8 , then any given chromosome that has an $80 \%$ or higher similarity with any other chromosome in the population will be removed based on a fitness-knock-out system (i.e. fitter chromosome is kept), and a random structure from the structure library will be used to replace the gap that is left.

From Figure 3a you can see that 1 and 0.8 provided more acceptable average twin removal levels. Both 0.6 and 0.4 , on the other hand, provided twin removal results that were too high for our GA to maintain worthwhile genetic traits from one generation to the next. Therefore, in essence the search becomes completely random, and the optimal solution is very unlikely to be found.

In Figure 3a the CCF value 0.8 increases slightly from 1, but this is not the case for 0.6 which takes a significant jump to an average twin removal percentage of $56.405 \%$. This is far from the optimal range presented in Hoque's work $(20 \%$ 40\%) using the HP low-resolution model. Even 1 and 0.8 using our approach achieved only $5.3295 \%$, and $6.032 \%$ average twin removal rates respectively. This directly implies that not enough highly-correlated chromosomes are being removed, and by having a higher twin removal percentage could lead to better solutions. The main cause for this could be due to the upper and lower bounds assumptions for the RMSD measure ( 0 for identical and 100 for completely different), if these values are not reflecting a proper 0 to $100 \%$ similarity between two protein structures then the average twin removal percentages may not be reflecting a true CCF value of $1,0.8$, 0.6 , and 0.4 .

To further quantify the performance of different $\mathrm{CCF}$ values with our approach, we looked at which $\mathrm{CCF}$ value provided better results. The results of all the proteins we tested for this can be found in Table II. For each protein in Table II a light grey highlight has been used to display the CCF value that produced the best result. In Figure $3 b$ we have condensed these results to display the average RMSD and TMScore improvements for a particular CCF value. These results corroborate with our average twin removal percentage results.

Figure $3 \mathrm{~b}$ clearly shows that by using no twin removal (WT) or using 1 and $0.8 \mathrm{CCF}$ values, on average, better solutions are found when compared to 0.6 and $0.4 \mathrm{CCF}$ values. This is depicted by WT, 1 , and 0.8 all having considerable average improvements for both RMSD and TM-score structural similarity measures. Compare this to 0.6 which has a small average improvement for TM-Score, and a large average deterioration in the RMSD compared to the best solution in the initial population, and 0.4 which has a average deterioration in both the RMSD and the TM-Score. This further confirms that both 0.6 and 0.4 are not keeping good genetic traits from one generation to the next making the GA act as a random search. Another point to mention is that to remove all bias from our results we have excluded any chromosome that is a direct copy from the structure library, therefore all results are structures that have been modified by our algorithm.

To show the validity of our twin-removal approach we created a Monte Carlo (MC) method and ran it for the same number of operations as our Genetic Algorithm (GA). The results for this can be found in Figures $4 a$ and $4 b$. The improvement percentages for the GA approach are taken from the best performing run with a particular CCF value (see Table II), and the improvement calculations for the MC approach are calculated based on how much the structure improved from its original state after the 16000 steps. From Figures $4 \mathrm{a}$ and $4 \mathrm{~b}$ you can see that the MC method performed poorly 
TABLE II: Comparison between different CCF values while performing twin removal (TR-WT means no twin removal used, TR-100 $=$ CCF of 1 , TR- $80=$ CCF of $>=0.8$, TR- $60=$ CCF of $>=0.6$, and TR-40 $=$ CCF of $>=0.4$.). The best result for each protein is highlighted in grey.

\begin{tabular}{|c|c|c|c|c|c|c|}
\hline Protein & $T R$ & $f$ & $R M S D$ & $T M$ & RMSD Improvement & TM-Score Improvement \\
\hline 2 ptl & WT & -100.92 & $4.405 \AA$ & 0.5470 & $49.74 \%$ & $33.94 \%$ \\
\hline $2 \mathrm{ptl}$ & 100 & -105.39 & $4.192 \AA$ & 0.5571 & $52.17 \%$ & $36.41 \%$ \\
\hline 2 ptl & 80 & -118.97 & $3.755 \AA$ & 0.5786 & $57.15 \%$ & $41.67 \%$ \\
\hline 2 ptl & 60 & -53.96 & $5.718 \AA$ & 0.4143 & $34.76 \%$ & $1.44 \%$ \\
\hline $2 \mathrm{ptl}$ & 40 & -88.02 & $6.320 \AA$ & 0.4437 & $27.89 \%$ & $8.64 \%$ \\
\hline 1pgx & WT & -86.16 & $2.954 \AA$ & 0.6752 & $32.63 \%$ & $2.77 \%$ \\
\hline $1 \operatorname{pgx}$ & 100 & -91.32 & $3.329 \AA$ & 0.6620 & $24.08 \%$ & $0.76 \%$ \\
\hline $1 \operatorname{lpg} x$ & 80 & -56.48 & $3.060 \AA$ & 0.6460 & $30.22 \%$ & $-1.67 \%$ \\
\hline 1pgx & 60 & -95.29 & $4.672 \AA ̊$ & 0.5293 & $-6.55 \%$ & $-19.44 \%$ \\
\hline 1pgx & 40 & -95.73 & $6.095 \AA$ & 0.4532 & $-39.00 \%$ & $-31.02 \%$ \\
\hline $1 \mathrm{bds}$ & WT & -19.49 & $6.355 \AA$ & 0.2273 & $-3.32 \%$ & $6.31 \%$ \\
\hline $1 \mathrm{bds}$ & 100 & 1.33 & $5.438 \AA$ & 0.3450 & $11.59 \%$ & $61.37 \%$ \\
\hline $1 \mathrm{bds}$ & 80 & -24.65 & $6.006 \AA$ & 0.2143 & $2.36 \%$ & $0.23 \%$ \\
\hline $1 \mathrm{bds}$ & 60 & -28.87 & $5.865 \AA$ & 0.2656 & $4.65 \%$ & $24.23 \%$ \\
\hline $1 \mathrm{bds}$ & 40 & -17.82 & $7.133 \AA$ & 0.2287 & $-15.96 \%$ & $6.97 \%$ \\
\hline $1 \mathrm{bm} 8$ & WT & -82.09 & $7.814 \AA$ & 0.2751 & $0.00 \%$ & $0.00 \%$ \\
\hline $1 \mathrm{bm} 8$ & 100 & -62.10 & $7.822 \AA$ & 0.2751 & $-0.10 \%$ & $0.00 \%$ \\
\hline $1 \mathrm{bm} 8$ & 80 & -66.01 & $7.444 \AA$ & 0.2816 & $4.74 \%$ & $2.36 \%$ \\
\hline $1 \mathrm{bm} 8$ & 60 & -75.66 & $7.630 \AA$ & 0.2940 & $2.35 \%$ & $6.87 \%$ \\
\hline $1 \mathrm{bm} 8$ & 40 & -75.66 & $8.040 \AA ̊$ & 0.2822 & $-2.89 \%$ & $2.58 \%$ \\
\hline $1 \mathrm{emw}$ & WT & -38.98 & $9.383 \AA$ & 0.3763 & $-11.50 \%$ & $31.76 \%$ \\
\hline $1 \mathrm{emw}$ & 100 & -56.69 & $7.893 \AA$ & 0.3795 & $6.20 \%$ & $32.88 \%$ \\
\hline 1emw & 80 & -46.67 & $8.525 \AA$ & 0.4123 & $-1.30 \%$ & $44.36 \%$ \\
\hline $1 \mathrm{emw}$ & 60 & -30.55 & $9.193 \AA$ & 0.3070 & -9.25 & $7.49 \%$ \\
\hline $1 \mathrm{emw}$ & 40 & -37.65 & $9.381 \AA$ & 0.2479 & $-11.48 \%$ & $-13.20 \%$ \\
\hline 1aoy & WT & -60.19 & $5.752 \AA$ & 0.5530 & $4.58 \%$ & $43.49 \%$ \\
\hline 1aoy & 100 & -64.57 & $5.692 \AA ̊$ & 0.5554 & $5.57 \%$ & $44.11 \%$ \\
\hline 1aoy & 80 & -44.39 & $3.967 \AA$ & 0.5235 & $34.19 \%$ & $35.83 \%$ \\
\hline 1aoy & 60 & -77.41 & $6.846 \AA$ & 0.5220 & $-13.57 \%$ & $35.44 \%$ \\
\hline 1aoy & 40 & -72.91 & $6.865 \AA$ & 0.4799 & $-13.89 \%$ & $24.52 \%$ \\
\hline $1 \mathrm{csp}$ & WT & -91.24 & $2.579 \AA$ & 0.7191 & -0.12 & $0.49 \%$ \\
\hline $1 \mathrm{csp}$ & 100 & -71.68 & $2.538 \AA$ & 0.7249 & $1.48 \%$ & $1.30 \%$ \\
\hline $1 \mathrm{csp}$ & 80 & -89.44 & $2.944 \AA$ & 0.6739 & $-14.29 \%$ & $-5.83 \%$ \\
\hline $1 \mathrm{csp}$ & 60 & -98.94 & $3.936 \AA$ & 0.5652 & $-52.80 \%$ & $-21.02 \%$ \\
\hline $1 \mathrm{csp}$ & 40 & -80.72 & $4.503 \AA$ & 0.4594 & $-74.81 \%$ & $-35.80 \%$ \\
\hline $2 p p p$ & WT & -23.94 & $8.765 \AA$ & 0.3776 & $9.00 \%$ & $35.83 \%$ \\
\hline $2 p p p$ & 100 & -38.76 & $9.011 \AA$ & 0.3226 & $6.45 \%$ & $16.04 \%$ \\
\hline $2 p p p$ & 80 & -34.24 & $8.992 \AA$ & 0.5156 & $6.64 \%$ & $85.47 \%$ \\
\hline $2 p p p$ & 60 & -43.99 & $9.122 \AA$ & 0.4464 & $5.29 \%$ & $60.58 \%$ \\
\hline $2 p p p$ & 40 & -26.03 & $11.321 \AA$ & 0.2605 & $-17.54 \%$ & $-6.29 \%$ \\
\hline $1 \mathrm{kjs}$ & WT & -41.81 & $4.214 \AA$ & 0.4926 & $7.20 \%$ & $4.67 \%$ \\
\hline $1 \mathrm{kjs}$ & 100 & -28.49 & $4.189 \AA$ & 0.4921 & $7.75 \%$ & $4.57 \%$ \\
\hline $1 \mathrm{kjs}$ & 80 & -36.20 & $4.173 \AA$ & 0.4827 & $8.10 \%$ & $2.57 \%$ \\
\hline $1 \mathrm{kjs}$ & 60 & -34.89 & $5.673 \AA$ & 0.3628 & $-24.93 \%$ & $-22.91 \%$ \\
\hline $1 \mathrm{kjs}$ & 40 & -41.98 & $4.941 \AA$ & 0.4111 & $-8.81 \%$ & $-12.64 \%$ \\
\hline $1 \mathrm{vec}$ & WT & -71.42 & $3.242 \AA$ & 0.7201 & $-9.90 \%$ & $5.90 \%$ \\
\hline $1 \mathrm{vcc}$ & 100 & -55.01 & $3.623 \AA$ & 0.6369 & $-22.81 \%$ & $-6.34 \%$ \\
\hline $1 \mathrm{vcc}$ & 80 & -47.45 & $2.937 \AA$ & 0.7271 & $-0.44 \%$ & $6.93 \%$ \\
\hline $1 \mathrm{vcc}$ & 60 & -58.35 & $6.270 \AA ̊$ & 0.5980 & $-112.54 \%$ & $-12.06 \%$ \\
\hline $1 \mathrm{vcc}$ & 40 & -58.02 & $5.829 \AA$ & 0.3697 & $-97.59 \%$ & $-45.63 \%$ \\
\hline
\end{tabular}




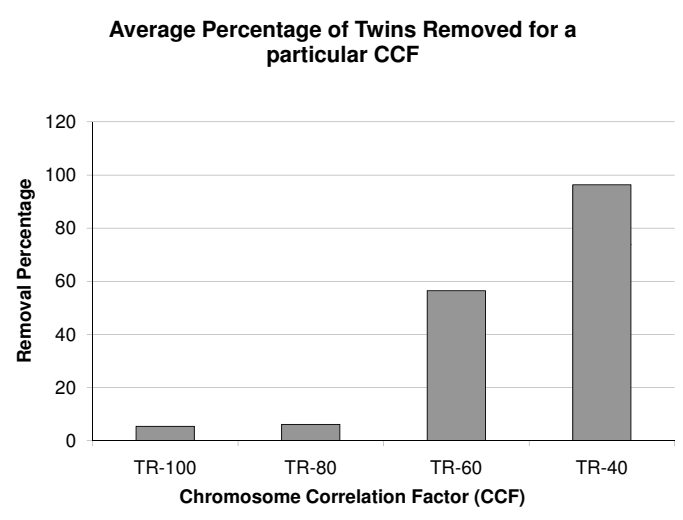

(a) Average Twins Removed

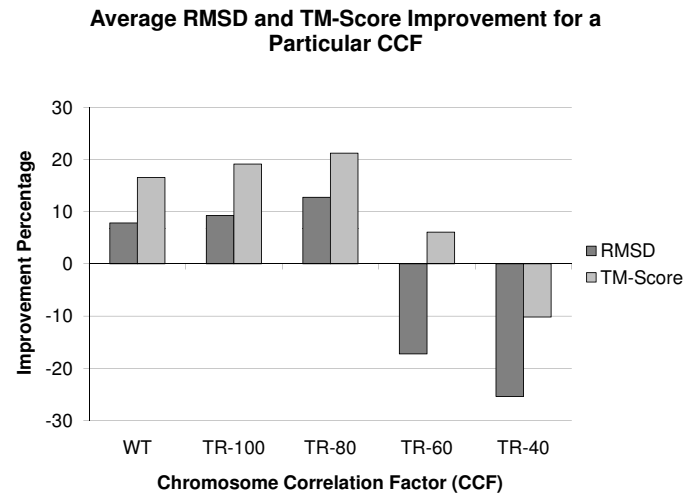

(b) Average Improvement

Fig. 3: (a) Shows the average percentage of twins removed for a particular CCF, and (b) Depicts the average RMSD and TM-Score improvement (in percentage) for a particular CCF $(\mathrm{TR}-100=\mathrm{CCF}$ of 1 , TR- $80=\mathrm{CCF}$ of $>=0.8$, TR-60 $=\mathrm{CCF}$ of $>=0.6$, and TR $-40=\mathrm{CCF}$ of $>=0.4$ )

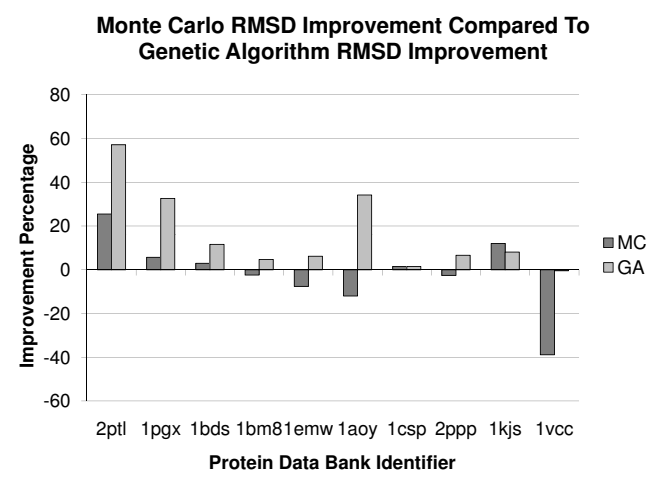

(a) RMSD

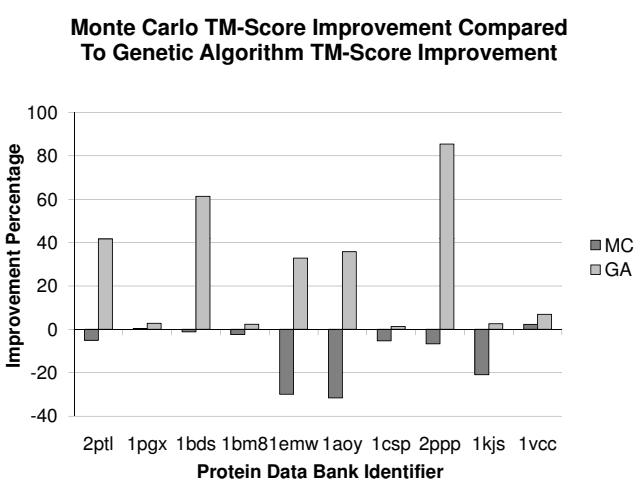

(b) TM-Score

Fig. 4: (a) Shows the TM-Score improvement percentages for our Monte Carlo and Genetic Algorithm methods, and (b) Shows the RMSD improvement percentages for our Monte Carlo and Genetic Algorithm methods.

when compared to our GA using twin removal techniques. On average there is no improvement to the original structure that the algorithm started with. Instead there is usually a deterioration from its original state (negative improvement). Figure 5 graphically depicts our improvements, for protein 1aoy, over our MC approach.

In the end the CCF value that provided us with the best average twin removal percentage and results was 0.8. It gave us an average $12.74 \%$ RMSD improvement and an average 21.19\% TM-Score improvement (See Figure 3b). This clearly demonstrates that twin removal protocols improve the GA search. For example, in Figure $3 \mathrm{~b}$ the average difference between using twin removal and not using twin removal, for the CCF value of 0.8 , is $4.91 \%$ for RMSD and $4.67 \%$ for TMScore. This difference can be quite considerable when trying to predict a protein's final three-dimensional conformation. We also demonstrated that by using twin removal techniques on our GA we could increase the performance of the search to be considerably better than the MC method.

\section{CONCLUSION}

Twins or identical chromosomes are a serious problem in Genetic Algorithms (GA) when solving hard optimisation problems. Identical chromosomes occur due to the population growing in similarity after each generation. This is even worse for optimisation problems like protein structure prediction (PSP) where a large amount of iterations are required to get a meaningful solution To limit the amount of twins present in the GA population a chromosome correlation factor (CCF) can be defined for twin removal. The innovation behind this idea was to remove not only identical chromosomes from the population, but to also remove highly-correlated chromosomes from the population.

In this paper we present a new twin removal protocol. To do this we made assumptions for the upper and lower bounds 


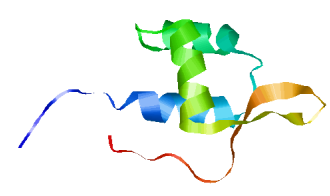

(a) Native

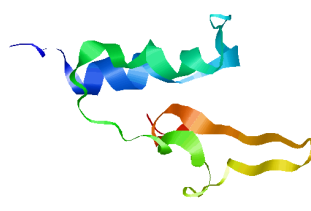

(b) MC

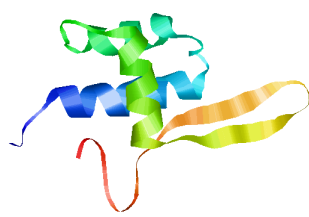

(c) GA

Fig. 5: Improvements for protein 1aoy, compared to native (a), best MC Model (b), and GA 80\% twin removal (c).

of the RMSD measure ( 0 for identical and 100 for completely different) to be able to define the structural similarity between two structures as a CCF value. We also developed a structure library of pre-built protein conformations to be used to fill in the gaps left by twins that had been removed from the population. This structure library was made up of protein structures that were generated by ab initio runs using Rosetta, and structures were pulled out of it at random.

By applying this approach to our GA we obtained results that were considerably better than when no twin removal techniques were used. This was clearly demonstrated in our results for the CCF value of 0.8 , which gave us an average $12.74 \%$ RMSD improvement and an average $21.19 \%$ TMScore improvement. For both average twin removal percentage and results the CCF value of 0.8 performed the best when compared to the other CCF values we tested. Also, in contrast to the HP low-resolution model, which obtained an average twin removal of $20 \%-40 \%$, our optimal CCF value produced an average twin removal of $6.032 \%$. We also demonstrated that by applying twin removal techniques to our GA we could obtain results that were significantly better than using a MC approach.

In regards to future work testing out different upper bounds for the RMSD measure could provide some insight into a more optimal setting than 100 to tell if two proteins share no structurally similar traits. Another possibility is to use different structural measures that have their upper and lower bounds already defined, such as TM-Score (between 0 and 1). Finally the twin removal process could be enhanced by generating structures for twin replacements in parallel with the prediction process.

\section{REFERENCES}

[1] C. Anfinsen, "Principles that govern folding of protein chains," Science, vol. 181, pp. 223-230, 1973.

[2] S. Wu, J. Skolnick, and Y. Zhang, "Ab initio modeling of small proteins by iterative TASSER simulations," BMC Biology, vol. 5, no. 17, 2007, doi:10.1186/1741-7007-5-17.

[3] A. Shmygelska and H. Hoos, "An ant colony optimisation algorithm for the 2D and 3D hydrophobic polar protein folding problem," $B M C$ Bioinformatics, vol. 6, no. 30, 2005.

[4] L.-H. Hung, S.-C. Ngan, T. Liu, and R. Samudrala, "PROTINFO: new algorithms for enhanced protein structure predictions," Nucleic Acids Research, vol. 33, pp. 77-80, 2005.

[5] E. Bornberg-Bauer, "Chain growth algorithms for HP-type lattice proteins," in Research in Computational Molecular Biology RECOMB, 1997, pp. 47-55.

[6] L. Toma and S. Toma, "Contact interactions methods: A new algorithm for protein folding simulations," Protein Science, vol. 5, no. 1, pp. 147153, 1996.
[7] V. Cutello, G. Nicosia, P. Pavone, and J. Timmis, "An immune algorithm for protein structure prediction on lattice models," IEEE Transactions on Evolutionary Computation, vol. 11, no. 1, pp. 101-117, 2007.

[8] M. Judy, K. Ravichandran, and K. Murugesan, "A multi-objective evolutionary algorithm for protein structure prediction with immune operators," Computer Methods in Biomechanics and Biomedical Engineering, vol. 12, no. 4, pp. 407-413, 2009.

[9] R. Unger and J. Moult, "Genetic algorithms for 3d protein folding simulations," Journal of Molecular Biology, vol. 231, pp. 75-81, 1993.

[10] T. Hoque, M. Chetty, and A. Sattar, "Extended HP model for protein structure prediction," Journal of Computational Biology, vol. 16, pp. $85-103,2009$.

[11] B. Blum, "Resampling methods for protein structure prediction," Ph.D. dissertation, Electrical Engineering and Computer Sciences University of California at Berkeley, Dec. 2008.

[12] F. Liang and W. Wong, "Evolutionary monte carlo for protein folding simulations," Journal of Chemical Physics, vol. 115, no. 7, pp. 33743380, 2001.

[13] T. Hoque, M. Chetty, A. Lewis, and A. Sattar, "Twin removal in genetic algorithms for protein structure prediction using low resolution model," IEEE/ACM Transactions on Computational Biology and Bioinformatics, 2009, doi:10.11.09/TCBB.2009.34.

[14] K. Simons and et al., "Prospects for ab initio protein structural genomics," Journal of Moleculer Biology, vol. 306, pp. 1191-1199, 2001.

[15] N. Metropolis and S. Ulam, "The monte carlo method," Journal of the American Statistical Association, vol. 44, pp. 335-341, 1949.

[16] T. Jiang, Q. Cui, G. Shi, and S. Ma, "Protein folding simulations of hydrophobic-hydrophilic model by combining tabu search with genetic algorithms," Journal of Chemical Physics, vol. 119, no. 8, pp. 45924596, 2003.

[17] J. Pedersen and J. Moult, "Protein folding simulations with genetic algorithms and a detailed molecular description," Journal of Molecular Biology, vol. 269, pp. 240-259, 1997.

[18] J. Arunachalam, V. Kanagasabai, and N. Gautham, "Protein structure prediction using mutually orthogonal latin squares and a genetic algorithm," vol. 342, pp. 424-443, 2006.

[19] S. Ronald, "Duplicate genotypes in a genetic algorithm," in IEEE World Congress on Computational Intelligence, 1998, pp. 793-798.

[20] R. Haupt and S. Haupt, Practical Genetic Algorithms - 2nd ed. Wiley, 2004.

[21] L. Eshelman and J. Schaffer, "Preventing premature convergence in genetic algorithms by preventing incest," in The 4th International Conference on Genetic Algorithms, 1991, pp. 115-122.

[22] K. Deb and S. Agrawal, "A niched-penalty approach for constraint handling in genetic algorithms," in Artifical Neural Nets and Genetic Algorithms, 1999, pp. 235-243.

[23] T. Higgs, B. Stantic, T. Hoque, and A. Sattar, "Genetic algorithm feature-based resampling for protein structure prediction," in IEEE World Congress on Computational Intelligence, 2010, pp. 2665-2672.

[24] V. Maiorov and G. Crippen, "Significance of root-mean-square deviation in comparing three-dimensional structures of globular proteins," Journal of Molecular Biology, vol. 235, pp. 625-634, 1994.

[25] Y. Zhang and J. Skolnick, "Scoring function for automated assessment of protein structure template quality," Proteins, vol. 57, pp. 702-710, 2004.

[26] — , "Tertiary structure predictions on a comprehensive benchmark of medium to large size proteins," Biophysical Journal, vol. 87, pp. 26472655, Oct. 2004. 